\title{
Authors Index
}

Numbers refer to Pages

AlACH M, 9 AKKERMANJWN, 199 ANDRE P, 55, ANDERSON K, 285 APITZ R, 46 AZNAR J, 166, 171 AZZAM , 55

BADIMON J, 269 BAL DIT SOLLIER C, 55 BARROCL1FFE TW, 266 BELLOCF, 182 BERGQVIST D, 378 BERTI F, 28 BOFFA MC, 233 BOISSEAUMR, 177, 182 BONEU B, 367 BONNEAU M, 55 BOSCH V, 46

CARMELIET P, 132 CARMENAR, 171, CARRERAS L.O, 339 CERLETTI C, 20 CHESEBRO J, 269 CLOSSEC, 177, 182, COLLEN D, 132, 293 COLLER B, 285 COLLET JP, 314 CONARDJ, 314

DAHLBACK B, 300 DAIMAUJ, 166 de BOSCH N, 46 de GAETANO G, 20 DEJANAE, 210 deIMASCHIOA, 210 DROUET L, 55

ELALAMYI, 314 ELDOR A, 37 EVANGELISTA V, 20

FALLON J, 269 FOLCO F, 28 FORAST1ERO RR, 339 FOXJ, 102

FRANSSEN J, 89 FUSTER V, 269

GANDRILLE S, 9 GILL, 171

HAINAUD P, 55 HELLGREN M, 244 HILLARPA, 300 HIRSH, 189

horelloumh, 314 houghton d, 266

Ireland h, 227

jespersen j, 266

KORNER M, 37 KYRIAKOULIS K, 227

LABIOSM, 166 LANE DA, 227 LINDHOUT T, 89 LLUCHI, 171

MACLOUF J, 28

MANNUCCI , 1

MARTI, 171

MARTINEZ M, 166, 171

MAURELM, 182

MULLER PEDDINGHAUS R, 28

NEMERSON Y, 98

NURDEN A, 78

PANET A, 37 PAYSANT J, 330 PICK M, 37

PIGNAUD G, 55 POLLER L, 266 PRUVOSTA, 177, 182

RENARDM, 177, 182 RESNICK-ROGUEL N, 37 ROSSONI G, 28

SALAA, 28 SALEMINK 1,89 SAMAMAMM, 314 SANDSETP, 154 SEIGNEUR M, 177, 182 SELA-DONENFELD D, 37 SIMON D, 314 SORIA C, 330 SORIA J, 330

TRIPLETT DA,357 TRIPODIA, 1,266 TROSSAERTM, 314 TURPIE A, 220

VALENTIN S, 89 van de WERF F, 293 van den BESSELAAR A, 248,266 van WILLIGEN G, 199 VANDERSCHUEREN S, 293 VANNIERJP, 314 VASSEM, 314 VAYA-MONTANA A, 166, 171 VERSTRAETE M, 70

WEISMAN H, 285 WILLEMS GM, 89 
ZANETTIA, 210 ZOLLER B, 300 